\title{
Batching Work and Rework Processes with Limited Deterioration of Reworkables
}

\author{
Karl Inderfurth ${ }^{a}$, Adam Janiak $^{b}$, Mikhail Y. Kovalyov ${ }^{c}$, Frank Werner ${ }^{a, 1}$ \\ ${ }^{a}$ Otto-von-Guericke-Universität, Magdeburg, Germany, E-mail: \\ inderfurth@ww.uni-magdeburg.de,frank.werner@mathematik.uni-magdeburg.de \\ ${ }^{b}$ Institute of Engineering Cybernetics, Wroclaw University of Technology, E-mail: \\ janiak@ict.pwr.wroc.edu.pl \\ ${ }^{c}$ Faculty of Economics, Belarus State University, and United Institute of Informatics \\ Problems, National Academy of Sciences of Belarus, E-mail: kovalyovmy@bsu.by
}

\begin{abstract}
We study the problem of planning the production of new and recovering defective items of the same product manufactured on the same facility. Items of the product are produced in batches. The processing of a batch includes two stages. In the first work stage, all items of a batch are manufactured and good quality items go to the inventory to satisfy given demands. In the second rework stage, some of the defective items of the same batch are reworked. Each reworked item has the required good quality. During waiting for rework, defective items deteriorate. There is a given deterioration time limit. A defective item that is decided not to be reworked or cannot be reworked because it will exceed the deterioration time limit is disposed of immediately after its work operation completes. Deterioration results in an increase in time and cost for performing rework processes. It is assumed that the percentage of defective items is the same in each batch, and that they are evenly distributed in each batch. A setup time as well as a setup cost is required to start batch processing and to switch from production to rework. The objective is to find batch sizes and positions of items to be reworked such that a given number of good quality items is produced and total setup, rework, inventory holding, shortage and disposal cost is minimized. A polynomial dynamic programming algorithm is presented to solve this problem.
\end{abstract}

Key Words: inventory control, batching, rework, deterioration, dynamic programming.

\footnotetext{
${ }^{1}$ Corresponding author
} 


\section{Introduction}

The problem of optimal planning work and rework processes belongs to the broad field of reverse logistics which deals with all kinds of reuse processes in supply chains, see de Brito and Dekker [1]. These processes aim to recover defective or used product items in such a way that they meet the quality level of a good item. The benefits are regaining the material and value added and improving the environment protection.

In many production situations, work and rework processes are performed on the same equipment. Flapper and Jensen [4] and Inderfurth and Teunter [8] note that such an integration leads to challenging planning and control problems.

Quite often reworkable items undergo a deterioration process while waiting to be recovered. This effect is especially important in process industries as indicated by Flapper et al. [2]. The deterioration of work-in-process results in an increase in processing time and cost for reworking the respective items.

In this paper, we address the following problem of scheduling work and rework processes. A single facility is used for manufacturing $N$ good quality items of the same product and reprocessing some of the defective items. One unit of time is required for manufacturing one item. Due dates $d_{j}, j=1, \ldots, N$, are given such that $j$ good quality items should ideally be manufactured by time $d_{j}$. Due dates can be obtained from demand planning because there is an evident relation between the due dates and demands for good quality items over time. We can assume without loss of generality that $d_{1} \leq \cdots \leq d_{N}$.

Product items are manufactured in batches. Batch processing may include two stages that we call work and rework. A setup time $s_{1}$ precedes the processing of each batch. During work stage, good quality as well as defective items are produced. We assume that the percentage of the defective items is given and that they are evenly distributed in each batch. Furthermore, we make a simplifying but still realistic assumption that in each batch the number of good quality items is a multiple of the number of defective items. Thus, we assume that each batch includes an integer number of blocks where each block consists of $v-1, v-1 \geq 1$, good quality items followed by a single defective item.

The work stage is completed when it is decided to start reprocessing of at least one defective item of the batch or $N$ good quality items have been produced. In the former case, a setup time $s_{2}$ is needed to switch from work to rework stage. Only those defective 
items can be reworked whose waiting time for rework does not exceed a given deterioration time limit $L$. After an age of $L$ time units it is assumed that deteriorating items are no longer recoverable or can only be reworked at a prohibitive cost. Some (or even all) defective items can be decided not to be reworked. A defective item that is decided not to be reworked or cannot be reworked is disposed of immediately after its work operation completes. Here we call processes of manufacturing and remanufacturing an item as work and rework operations, respectively. After remanufacturing, defective items are in the state of good quality items.

The above description shows that processing of every batch with a possible exception for the last batch includes two stages. The last batch may contain no reworked item.

We assume that the facility is an expensive equipment and, therefore, there is no idle time from time zero until $N$ good quality items have been produced.

We separate from a trivial case when $s_{2}>L$. In this case, no defective item can be reworked and there is only one feasible schedule that consists of a single batch. In the sequel, $s_{2} \leq L$ is assumed.

Since $N$ good quality items have to be produced in blocks and there are $v-1$ good quality items in each block, the total number of blocks to be scheduled does not exceed $n:=\lceil N /(v-1)\rceil$, where $\lceil a\rceil$ is the smallest integer greater than or equal to $a$.

A batch setup cost is associated with each batch. Furthermore, there is a holding cost for each time unit of a good quality item to be in inventory, a holding cost for each time unit of a reworkable item to await rework, a disposal cost for a non-reworked defective item and a shortage cost for each time unit passed between the completion of $j$ good quality items and the due date $d_{j}$ if $j$ th good quality item is produced after $d_{j}, j=1, \ldots, N$.

Due to deterioration, the per-item rework time and cost is often not constant. Even if it increases linearly with the time that a reworkable item waits for reprocessing, the corresponding model is still too complicated to find an optimal solution in a reasonable time, see Inderfurth et al. [7] and Inderfurth et al. [6]. Therefore, like it was done in the above mentioned papers, we make the following reasonable simplification of the deterioration model.

Let $R(y)$ denote the total processing time of $y$ rework operations in the same batch. Assume that $R(y)$ is a linear function of $y$. This assumption is motivated by the fact that 
the average waiting time of defective items to be reworked is proportional to their average stock. Thus, the individual deterioration of reworkable items in a batch is replaced by the batch deterioration that is assumed to be linear. It should be noted that hereby the impact of sequencing individual rework operations on the rework operation processing time is not specifically taken into consideration. Let $R(y)=A+B y$. To minimize deviation from the situation with individual deterioration of reworkable items, we assume that the processing time of a rework operation that starts as $j$ th among $y$ rework operations in the same batch is equal to $p_{j}=p+j \delta(y), j=1, \ldots, y$. Here $p$ stands for a basic rework processing time and $\delta(y)$ describes the mean increase in rework time per item caused by waiting and deterioration. The value $\delta(y)$ can be found from $\sum_{j=1}^{y} p_{j}=A+B y$. We have

$$
\delta(y)=\frac{2 A+2 y(B-p)}{y(y+1)} .
$$

Given a batch with $b$ blocks and the same number of defective items, we assume that the positions of defective items are numbered $1,2, \ldots, b$ in order of their manufacturing. Every schedule is characterized by the number of batches, the number of blocks in each batch and the positions of defective items to be reworked in each batch. For example, a schedule can be represented by a sequence

$$
\left(b_{1},\left(i_{1}, \ldots, i_{x}\right)\right),\left(b_{2},\left(j_{1}, \ldots, j_{y}\right)\right)
$$

where $x \leq b_{1}$ and $y \leq b_{2}$. In this case, there are two batches with $b_{1}$ and $b_{2}$ blocks sequenced in this order. In the first batch, $x$ defective items in positions $i_{1}, \ldots, i_{x}$ are reworked in this order. Therefore, the remaining $b_{1}-x$ defective items of the first batch are disposed of. Similar information can be obtained for the second batch. The total number of good quality items produced according to this schedule is equal to $(v-1)\left(b_{1}+b_{2}\right)+x+y$.

Consider a feasible schedule. We say that a good quality item is labelled $j$ and $j$ is its label, if it is completed (manufactured or remanufactured) as $j$ th among good quality items. Furthermore, if we say that some item is labelled $j$, then we assume that this item is a good quality item completed as $j$ th. For formulating the cost function in our problem setting, the following notation is introduced.

$C_{j}$ - completion time of an item labelled $j$.

$S_{j}^{R}$ - start time of rework operation of an item labelled $j$ in case of rework.

$C_{j}^{W}$ - completion time of work operation of an item labelled $j$. 
$E$ - set of early good quality items such that $C_{j}<d_{j}$.

$T$ - set of tardy good quality items such that $C_{j}>d_{j}$.

$I$ - set of in-time good quality items such that $C_{j}=d_{j}$. We have $|E \cup T \cup I|=N$.

$G$ - set of reworked items. For a reworked item labelled $j$ we must have $S_{j}^{R}-C_{j}^{W} \leq L$.

$D$ - set of disposed defective items.

The following costs are considered:

$\alpha_{1}-$ batch setup cost,

$\alpha_{2}-$ good quality item holding cost (earliness cost),

$\alpha_{3}$ - good quality item shortage cost (tardiness cost),

$\alpha_{4}$ - reworkable item holding and rework cost,

$\alpha_{5}$ - defective item disposal cost.

Let $S$ be a schedule with $k$ batches and $N$ good quality items. The total cost of such a schedule is calculated as follows:

$$
F(S)=\alpha_{1} k+\alpha_{2} \sum_{j \in E}\left(d_{j}-C_{j}\right)+\alpha_{3} \sum_{j \in T}\left(C_{j}-d_{j}\right)+\alpha_{4} \sum_{j \in G}\left(S_{j}^{R}-C_{j}^{W}\right)+\alpha_{5}|D| .
$$

The problem is to find a schedule with minimum value $F(S)$. We denote this problem as WRLD, where the abbreviation can be read as work-rework-limited-deterioration.

Note that the batch setup cost can be different for a batch that includes no rework operation and for a batch that includes at least one rework operation. This difference can easily be incorporated into the model and handled by the solution method proposed below. However, a batch with no rework operation can occur only once at the end of the schedule. Therefore, we decided not to pay special attention to the case when the cost for the last batch can be different.

Furthermore, our solution method enumerates reworked items. Therefore, it can easily be adapted for the case when a fixed cost for each reworked item is included into the objective function.

There is a vast body of literature dealing with production and inventory control problems in case of product deterioration, see Goyal and Giri [5] for a recent survey. We also found a great amount of publications dealing with planning and control in case of rework, see Flapper and Jensen [4] for an overview. There are, however, very few publications addressing the problem area which combines both cases like it is done in our paper. A 
problem similar to problem WRLD was studied by Teunter and Flapper [12] and Flapper and Teunter [3]. They considered a situation where occurrence of a defective item is random and where demand is not restrictive insofar as every good quality item can be sold immediately. Inderfurth et al. [7] and Inderfurth et al. [6] addressed continuous and discrete versions, respectively, of the problem differing from problem WRLD in that the demand should always be satisfied and all defective items are reworked. In the continuous version [7], the demand rate was assumed to be constant and rework time and cost increased linearly with the batch size. The assumptions in the discrete version [6] were the same as in problem WRLD except that the deterioration was unlimited and no shortage was allowed. The latter differences make the results of [6] not applicable for problem WRLD. As far as we know, there are no results available in the literature for problem WRLD.

In the following section, we discuss the problem of determining an optimal sequence of items to be reworked in an arbitrary batch. In Section 3, we describe a dynamic programming algorithm for problem WRLD. It runs in $O\left(n^{5} N\right)$ time which is polynomial because there can be $N$ distinct due dates in the input and $n<N$. The paper concludes with a discussion of related problems and suggestions for future research.

\section{An optimal sequence of items to be reworked}

In the next section, we shall describe a dynamic programming algorithm for problem WRLD. In each iteration of this algorithm, a batch with given number $b$ of blocks and given number $y, 0 \leq y \leq b$, of defective items to be reworked is added to the end of a partial schedule. Here $y=0$ implies that the added batch is the last one and $N$ good quality items will be produced without remanufacturing any item of this last batch.

Let us first consider a situation when $1 \leq y \leq b$. Given a pair $(b, y)$, the problem appears whether this pair is feasible and, if it is feasible, which defective items have to be reworked and in which sequence. An example of a batch associated with a pair $(b, y)$ is given in Fig. 1.

Let a pair $(b, y)$ be fixed. Observe that the positions of defective items to be reworked affect only reworkable item holding and rework cost $\alpha_{4} \sum_{j \in G}\left(S_{j}^{R}-C_{j}^{W}\right)$. An optimal policy of determining these positions is established in the following theorem. 


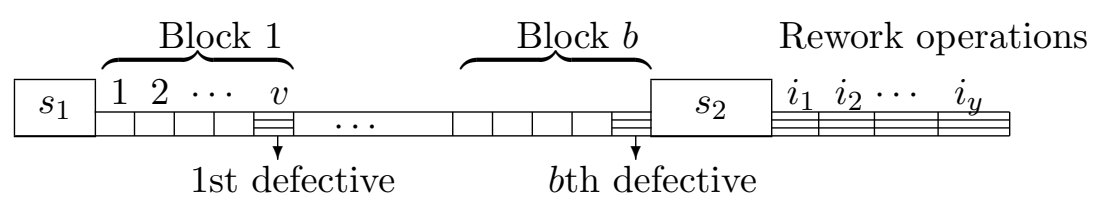

Figure 1: A batch with $b$ blocks and $y$ defective items in positions $i_{1}, i_{2}, \ldots, i_{y}$ to be reworked

Theorem 1 For problem WRLD, if a pair $(b, y)$ is fixed, then it is optimal to rework $y$ last defective items in the corresponding batch according to the policy "First come, first served" (FCFS), which is to rework $(b-y+j)$ th defective item as $j$ th, $j=1, \ldots, y$.

Proof. Given a pair $(b, y)$, let $\left(i_{1}, \ldots, i_{y}\right)$ be an optimal sequence of the positions of defective items to be reworked in this order. Consider this sequence.

Since the processing time of a rework operation sequenced as $j$ th is equal to $p+j \delta(y)$, $j=1, \ldots, y$, the holding time of the defective item in position $i_{j}$ to be reworked as $j$ th is equal to

$$
h_{i_{j}, j}=\left(b-i_{j}\right) v+s_{2}+(j-1) p+j(j-1) \delta(y) / 2
$$

We must have $h_{i_{j}, j} \leq L, j=1, \ldots, y$, to satisfy the deterioration time limit.

The contribution of the sequence $\left(i_{1}, \ldots, i_{y}\right)$ to the reworkable items holding and rework cost is equal to

$$
\begin{gathered}
\operatorname{HRC}\left(i_{1}, \ldots, i_{y}\right):=\alpha_{4} \sum_{j=1}^{y} h_{i_{j}, j}=\alpha_{4}\left[v b y-v \sum_{j=1}^{y} i_{j}+\right. \\
\left.y s_{2}+\frac{p y(y-1)}{2}+\left(\frac{y(y+1)(2 y+1)}{12}-\frac{y(y+1)}{4}\right) \delta(y)\right] .
\end{gathered}
$$

It is easy to see that the value of $H R C$ is independent of the sequence of indices $i_{1}, \ldots, i_{y}$. Furthermore, it is minimized when $i_{j} \in\{b-y+1, b-y+2, \ldots, b\}, j=1, \ldots, y$, i.e., when the last $y$ defective items are reworked.

Thus, any sequence of defective items in positions $b-y+1, b-y+2, \ldots, b$ feasible with respect to the deterioration time limit $L$ is optimal.

Assume that $i_{j}>i_{j+1}$ for $1 \leq j \leq y-1$, i.e., a defective item manufactured later is reworked earlier than the one manufactured earlier, see Fig. 2.

Then $h_{i_{j}, j}<h_{i_{j+1}, j+1} \leq L$. Interchange $i_{j}$ and $i_{j+1}$ in the sequence $\left(i_{1}, i_{2}, \ldots, i_{y}\right)$. Holding time for a defective item in position $i_{j+1}$ is decreased and holding time for a 


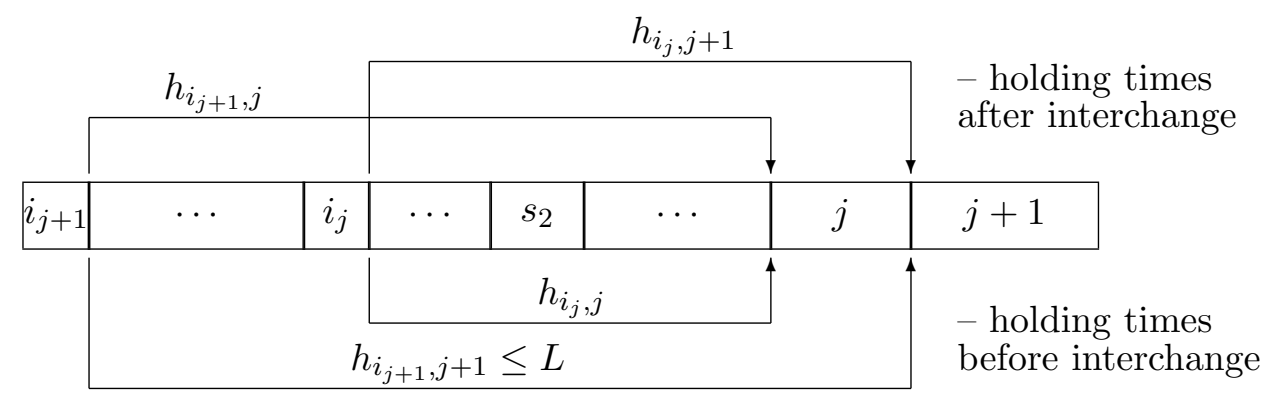

Figure 2: Positions of items $i_{j}$ and $i_{j+1}$ and their holding times

defective item in position $i_{j}$ is increased but it does not exceed $h_{i_{j+1}, j+1} \leq L$. Therefore, the new sequence is feasible and, as we have shown above, optimal.

The described interchange procedure can be applied a finite number of times to all items to be reworked that do not satisfy the policy FCFS and an optimal sequence satisfying this policy can finally be constructed.

The above proof shows that the sequence of defective items to be reworked affects only the number of such items that can fit into the deterioration time limit $L$ : the policy FCFS maximizes this number. Notice that this policy is reversed with respect to the policy "First come, last served" (FCLS) optimal for the problem with unlimited deterioration studied by Inderfurth et al. [6].

Theorem 1 implies that an optimal solution to problem WRLD is completely characterized by the sequence of pairs $\left(\left(b_{1}, y_{1}\right), \ldots,\left(b_{k}, y_{k}\right)\right)$, where $k$ is the number of batches, $b_{j}$ and $y_{j}$ are numbers of blocks and defective items to be reworked, respectively, in the batch sequenced $j$ th, $j=1, \ldots, k$. The defective items to be reworked are the last defective items in the batch and they are reworked according to the policy FCFS. Furthermore, we must have $(v-1) \sum_{i=1}^{k} b_{i}+\sum_{i=1}^{k} y_{i}=N$.

Given a pair $(b, y)$, its feasibility can be determined as follows. Calculate maximum holding time for items to be reworked:

$$
h_{\max }(y)=\max \{\mu(j) \mid j=1, \ldots, y\},
$$

where

$\mu(j)=(y-j) v+s_{2}+(j-1) p+\frac{j(j-1) \delta(y)}{2}=\frac{\delta(y)}{2} j^{2}-\left(v+\frac{\delta(y)}{2}-p\right) j+y v+s_{2}-p$ 
If $h_{\max }(y) \leq L$, then the pair $(b, y)$ is feasible. Otherwise, it is infeasible. Since function $\mu(j)$ is quadratic, $h_{\max }(y) \in\left\{\mu(1), \mu(y), \mu\left(\left\lfloor j^{*}\right\rfloor\right), \mu\left(\left\lceil j^{*}\right\rceil\right)\right\}$, where $j^{*}=\frac{v-p}{\delta(y)}+\frac{1}{2}$ is a solution of the differential equation $\mu^{\prime}(j)=\delta(y) j-\left[v-p+\frac{\delta(y)}{2}\right]=0$. Here $\left\lfloor j^{*}\right\rfloor$ is the integer part of $j^{*}$. Thus, $h_{\max }(y)$ can be calculated in $O(1)$ time.

Note that function $h_{\max }(y)$ is increasing in $y$. Therefore, by applying a bisection search in the range $y \in\{1, \ldots, n\}$ of possible values of $y$, we can find in $O(\log n)$ time value $y_{\max }$ such that either $y_{\max }=n$ and $h_{\max }(n) \leq L$ or $h_{\max }\left(y_{\max }\right) \leq L$ and $h_{\max }\left(y_{\max }+1\right)>L$.

In each iteration of the bisection search procedure, we determine in $O(1)$ time whether $h_{\max }\left(y^{0}\right) \leq L$ for a trial value $y^{0} \in\{V, V+1, \ldots, U\}$. We start with $V=1, U=n$ and $y^{0}=n$. Given $y^{0}$, if the indicated inequality is correct, then we reset $V:=y^{0}$. Otherwise, $U:=y^{0}$. In both cases, the next trial value is set to $\lfloor(V+U) / 2\rfloor$. The procedure terminates when $U \leq V+1$. In this case, we determine $y_{\max }=V$.

From now on, we assume that $y \in\left\{1, \ldots, y_{\max }\right\}$. All pairs $(b, y)$ such that $1 \leq y \leq$ $\min \left\{b, y_{\max }\right\}$ are feasible.

Denote by $h^{*}(y)$ the contribution of a feasible pair $(b, y)$ to the reworkable items holding and rework cost. Calculate

$$
\begin{gathered}
h^{*}(y)=\alpha_{4} \sum_{j=1}^{y}\left[(y-j) v+s_{2}+(j-1) p+\frac{j(j-1) \delta(y)}{2}\right]= \\
\alpha_{4}\left[\frac{v y(y-1)}{2}+y s_{2}+\frac{p y(y-1)}{2}+\left(\frac{y(y+1)(2 y+1)}{12}-\frac{y(y+1)}{4}\right) \delta(y)\right]= \\
\alpha_{4}\left[\frac{v y(y-1)}{2}+y s_{2}+\frac{p y(y-1)}{2}+\frac{(A+y(B-p)(2 y+1)}{6}-\frac{A+y(B-p)}{2}\right] .
\end{gathered}
$$

To unify presentation, set $h^{*}(0)=0$. Values $h^{*}(y), y=0,1, \ldots, y_{\max }$, can be calculated in $O(n)$ time.

\section{Dynamic programming algorithm}

We present a dynamic programming algorithm, denoted as DP, to solve problem WRLD. In this algorithm, batch schedules are constructed forwards starting from time zero. In each iteration, a single batch is added to the end of a current partial schedule. This batch includes a given number of blocks $b$ and a given number of items to be reworked $y$, $0 \leq y \leq \min \left\{b, y_{\max }\right\}$. If the added batch is not the last one, then $y \geq 1$. 
We say that a partial schedule is in the state $(k, r, x)$ if it includes $k$ batches, $r$ blocks and $x$ reworked items. Observe that all partial schedules in the same state complete at the same time

$$
C(k, r, x)=\left(s_{1}+s_{2}+A\right) k+v r+B x
$$

and have the same number of good quality items $(v-1) r+x$. Therefore, a schedule with minimum objective function value dominates all other schedules in the same state, that is, it can be extended to a complete feasible schedule for $N$ good quality items with minimum objective function value among all complete feasible schedules extended from partial schedules in the same state.

In algorithm DP, we recursively compute value $F(k, r, x)$, which is the minimum objective function value for feasible schedules in the state $(k, r, x)$.

If a batch associated with a feasible pair $(b, y)$ is added to the end of a schedule in the state $(k, r, x)$, then its contribution to the objective function, denoted as $\Delta(b, y)$, can be calculated as follows. Contributions to the batch setup cost, reworkable items holding and rework cost, and disposal cost are equal to $\alpha_{1}, \alpha_{4} h^{*}(y)$ and $\alpha_{5}(b-y)$, respectively.

Contribution of good quality items of this batch to the earliness and tardiness cost, denoted as $E T(b, y)$ can be calculated as follows. Calculate their completion times $C_{j}$, $j=J+1, J+2, \ldots, J+b(v-1)+y$, where $J=r(v-1)+x$ is the number of good quality items in the original schedule. We have

$$
C_{J+(i-1)(v-1)+q}=C(k, r, x)+s_{1}+(i-1) v+q, i=1, \ldots, b, q=1, \ldots, v-1
$$

for good quality items manufactured in the work stage and, if $y \geq 1$,

$$
C_{J+b(v-1)+u}=C(k, r, x)+s_{1}+b v+s_{2}+u p+u(u+1) \delta(y) / 2, u=1, \ldots, y
$$

for reworked items. Thus, completion times $C_{j}, j=J+1, \ldots, I+b(v-1)+y$, can be calculated in $O(b v+y)$ time.

Determine sets $E^{\prime}$ and $T^{\prime}$ of early and tardy items such that $j \in E^{\prime}$ if $C_{j}<d_{j}$, and $j \in T^{\prime}$ if $C_{j}>d_{j}, j=J+1, \ldots, J+b(v-1)+y$. Now we can calculate

$$
E T(b, y)=\alpha_{2} \sum_{j \in E^{\prime}}\left(d_{j}-C_{j}\right)+\alpha_{3} \sum_{j \in T^{\prime}}\left(C_{j}-d_{j}\right)
$$

and the contribution of the added batch to the objective function

$$
\Delta(b, y)=\alpha_{1}+E T(b, y)+\alpha_{4} h^{*}(y)+\alpha_{5}(b-y) .
$$


Time of calculating $\Delta(b, y)$ is determined by the time of calculating $E T(b, y)$. Therefore, it is equal to $O(b v+y)$.

A formal description of algorithm DP is given below. Recall that $n=\lceil N /(v-1)\rceil$.

\section{Algorithm DP}

Step 1 (Initialization) Set $F(k, r, x)=0$ for $(k, r, x)=(0,0,0)$ and $F(k, r, x)=\infty$ for $(k, r, x) \neq(0,0,0), k=1, \ldots, n, r=k, k+1, \ldots, n, x=k, k+1, \ldots, r$. Set $k=0$.

Step 2 (Recursion) For $r^{\prime}=k+1, \ldots, n$ and $x^{\prime}=k+1, \ldots, r^{\prime}$ such that $r^{\prime}(v-1)+x^{\prime} \leq N$ compute the following.

If $r^{\prime}(v-1)+x^{\prime}<N$, then

$$
F\left(k+1, r^{\prime}, x^{\prime}\right)=\min \left\{F(k, r, x)+\Delta(b, y) \mid b=1, \ldots, r^{\prime}, y=1, \ldots, x^{\prime}\right\}
$$

where $r=r^{\prime}-b$ and $x=x^{\prime}-y$. Set $k=k+1$ and repeat Step 2 .

If $r^{\prime}(v-1)+x^{\prime}=N$, then

$$
F\left(k+1, r^{\prime}, x^{\prime}\right)=\min \left\{F(k, r, x)+\Delta(b, y) \mid b=1, \ldots, r^{\prime}, y=0,1, \ldots, x^{\prime}\right\},
$$

where $r=r^{\prime}-b$ and $x=x^{\prime}-y$. Go to Step 3 .

Step 3 (Optimal solution) Calculate the optimal solution value

$$
\begin{gathered}
F^{*}=\min \{F(k, r, x) \mid k=1, \ldots, n, r=k, k+1, \ldots, n, \\
x=k, k+1, \ldots, r, r(v-1)+x=N\} .
\end{gathered}
$$

The corresponding optimal batch schedule can be found by backtracking.

It is easy to see that the time complexity of algorithm DP can be estimated as $O\left(n^{3} Q\right)$, where $Q$ is the time complexity of computing the minimum in the first recursive equation. This minimum can be found in $O\left(n^{2} N\right)$ time because $O(b v+y)=O(N)$ time is needed to calculate one value $\Delta(b, y)$ and there are $O\left(n^{2}\right)$ such values under the minimum. Thus, the overall time complexity of algorithm DP is $O\left(n^{5} N\right)$. 


\section{Related problems and further research}

In this section, we consider the following two problems related to problem WRLD.

Problem WRLD1. Let $f(b, y)$ denote the contribution of the first batch including $b$ blocks and $y$ defective items to be reworked to the objective function of problem WRLD. Let $t(b, y)$ denote the total setup and processing time of this batch. In problem WRLD1, the pair $(b, y)$ feasible with respect to the deterioration time limit $L$ should be determined such that the per time unit cost $f(b, y) / t(b, y)$ is minimized.

Such an approach to modelling situations in planning production with reworkable defectives is known in the literature, see for example, Liu and Yang [10] and Teunter and Flapper [12]. It represents some kind of myopic policy applied to the dynamic planning problem. In case of a stationary demand process and an unlimited planning horizon this policy is expected to provide a satisfactory solution.

Observe that $f(b, y)$ is equal to the value $\Delta(b, y)$ from the previous section calculated for the case when a batch associated with the pair $(b, y)$ is added to an empty schedule in the state $(k, r, x)=(0,0,0)$. Calculate

$$
t(b, y)=s_{1}+v b+s_{2}+A+B y
$$

An optimal solution to problem WRLD1, denoted as $\left(b^{*}, y^{*}\right)$, can be found from

$$
\frac{f\left(b^{*}, y^{*}\right)}{t\left(b^{*}, y^{*}\right)}=\min \left\{\frac{f(b, y)}{t(b, y)} \mid b=1, \ldots, n, y=0,1, \ldots, \min \left\{b, y_{\max }\right\}\right\}
$$

All required values $f(b, y)$ can be calculated in $O\left(n^{2} N\right)$ time, which is the time complexity of solving problem WRLD1 as well.

Problem Multi-WRLD. This problem differs from problem WRLD in that there are items of $Q, Q \geq 2$, products to be manufactured. Due dates $d_{j, q}$ are given such that $j$ good quality items of product $q$ should ideally be manufactured by $d_{j, q}, j=1, \ldots, N_{q}$, $q=1, \ldots, Q$, where $N_{q}$ is the total demand for good quality items of product $q$. As before, items are processed in batches. Each batch is preceded by a product dependent setup time. Batch processing includes work and rework stages separated by another product dependent setup time. Only items of the same product can form a batch. The objective is to find batch sizes and positions of items to be reworked such that the total demands 
$N_{1}, \ldots, N_{Q}$ are satisfied and total setup, rework, inventory holding, shortage and disposal cost is minimized.

Similar to [6], it is easy to demonstrate that problem Multi-WRLD is NP-hard in the strong sense if $Q$ is variable. It can be solved by a modification of algorithm DP in time polynomial in $N_{1}, \ldots, N_{Q}$ and exponential in $Q$. Therefore, it is polynomially solvable if $Q$ is a constant.

In problem WRLD, we assumed that the individual deterioration of reworkable items in a batch is replaced by the batch deterioration. Further research can be undertaken to find tractable variants of the original problem in which time and cost of reworking an individual defective item depends on its holding time.

In many practical situations, shortage (tardiness) costs are hard to quantify so that a service level approach is preferred. One realization of this approach is to use a time dependent upper bound on the number of tardy items. It can be analyzed whether problem WRLD is still tractable if a respective additional restriction is incorporated into the model.

In the multi-product case, the work-rework policy that a work stage is always followed by the rework stage can be relaxed. This restriction is adequate only for special cases of real work-rework processes, see Minner and Lindner [11]. In general, it has to be allowed that multiple rework stages on different products follow one manufacturing stage and vice versa.

\section{Acknowledgment}

This research was partially supported by INTAS under grant number 03-51-5501.

\section{References}

[1] de Brito M. and Dekker R. (2004) Reverse logistics: a framework. In: Dekker R., Fleischmann M., Inderfurth K. and van Wassenhove L. N. (eds.), Reverse Logistics Quantitative Models for Closed-Loop Supply Chains, Springer, 3-27.

[2] Flapper S.D.P., Fransoo J.C., Broekmeulen R.A.C.M. and Inderfurth K. (2002) Planning and control of rework in the process industries: a review, Production Planning \&6 Control 1 26-34. 
[3] Flapper S.D.P. and Teunter R.H. (2003) Logistic planning of rework with deteriorating work-in-process. Working paper. Department of Technology Management, Eindhoven University of Technology, The Netherlands.

[4] Flapper S.D.P. and Jensen T. (2003) Logistic planning and control of rework, International Journal of Production Research, to appear.

[5] Goyal S.K. and Giri B.C. (2001) Recent trends in modeling of deteriorating inventory, European Journal of Operational Research 134 1-16.

[6] Inderfurth K., Kovalyov M.Y., Ng C.T. and Werner F. (2003) Cost minimizing scheduling of work and rework processes on a single facility under deterioration of reworkables. Preprint 30/2003. Faculty of Mathematics, Otto-von-Guericke-University Magdeburg, Germany (accepted by International Journal of Production Economics).

[7] Inderfurth K., Lindner G. and Rahaniotis N.P. (2003) Lotsizing in a production system with rework and product deterioration. Preprint 1/2003. Faculty of Economics and Management, Otto-von-Guericke-University Magdeburg, Germany.

[8] Inderfurth K. and Teunter R.H. (2003) Production planning and control of closedloop supply chains. In: Guide Jr. V.D.R and van Wassenhove L.N. (eds.), Business perspectives on closed-loop supply chains, Carnegie Mellon University Press, 149-173.

[9] Lindner G., Buscher U. and Flapper S.D.P. (2001) An optimal lot and batch size policy for a single item produced and remanufactured on one machine. Working paper 10/2001. Faculty of Economics and Management, Otto-von-Guericke-University Magdeburg, Germany.

[10] Liu J.J. and Yang P. (1996) Optimal lot sizing in an imperfect production system with homogeneous reworkable jobs, European Journal of Operational Research 91 $517-527$.

[11] Minner S. and Lindner G. (2004) Lotsizing decisions in product recovery management. In: Dekker R., Fleischmann M., Inderfurth K. and van Wassenhove L.N (eds.), Reverse Logistics - Quantitative Models for Closed-Loop Supply Chains, Springer, 157-179.

[12] Teunter R.H. and Flapper S.D.P. (2003) Lot-sizing for a single-stage single-product production system with rework of perishable production defectives, OR Spectrum $\mathbf{2 5}$ 85-96. 